\title{
A case of myasthenia gravis treated with steroids: some suggestions for the mechanisms underlying their effect
}

\author{
P. DANDONA \\ M.R.C.P., D.Phil. \\ E. J. Corkan \\ M.B., B.S. \\ G. E. SLADEN \\ D.M., M.R.C.P.
}

Academic Division of Medicine, Royal Hospital, Sheffield

\begin{abstract}
Summary
This report deals with a patient with long standing myasthenia gravis treated with a small dose of corticosteroids. The response to treatment was prompt and the post-therapeutic deterioration in function was minimal and cholinergic in nature. It is suggested that the dose of anticholinesterases be reduced concomitantly with the institution of steroid treatment.
\end{abstract}

\section{Introduction}

Steroids and adrenocorticotrophic hormone (ACTH) have been used increasingly in the treatment of myasthenia gravis during the past few years, and this subject has been reviewed by Brunner, Namba and Grob (1972), Cape and Utterback (1972), Turner (1974) and Liversedge et al. (1974).

The prime indication for the use of steroids/ACTH in myasthenia is the progressive diminution in the efficacy of anticholinesterases (prostigmine and pyridostigmine) which develops in some patients. Two further indications are the inability of the cholinesterases to induce an initial remission in the symptoms and the overwhelming side effects caused by these drugs in some patients. In the following case report, we induced a remission with a low dose of prednisolone in a patient with severe chronic myasthenia who had very troublesome gastrointestinal side effects from anticholinesterases. Low dose prednisolone was employed in an attempt to minimize the post-therapeutic deterioration which is extremely common in these patients. During the course of treatment, we made some unexpected observations and their possible significance is discussed.

\section{Case report}

P.B. first presented, when aged 54 years, at the Royal Hospital, Sheffield, with hypothyroidism and a large tender goitre. Hashimoto's thyroiditis was

Correspondence: Dr P. Dandona, Royal Free Hospital, Pond Street, Hampstead, London NW3 2QG. diagnosed and she was treated with thyroid extract. Her symptoms subsided. Two years later she presented with generalized weakness, fatiguability, dysphagia, dysarthria and ptosis. A diagnosis of myasthenia gravis was made. On treatment with prostigmine, her symptoms remitted; however, during the subsequent months, the dose of prostigmine had to be increased, each increment being associated with increasing abdominal cramp, nausea, vomiting and diarrhoea. Atropine was added to treatment. During the next 10 years the myasthenic symptoms gradually progressed, and diuretics were then added to her treatment because she had mild congestive cardiac failure attributed to ischaemic heart disease. One year later, prostigmine had become ineffective and pyridostigmine was substituted. Weakness progressed in spite of $960 \mathrm{mg}$ of pyridostigmine per day and any further increase in its dose was not possible because of gastrointestinal side effects.

When seen at the age of 70 years she had gross ptosis and had to part her eyelids with her hands in order to see who was examining her. Her head constantly fell forwards and the provision of a collar had not helped. There was gross diplopia, bilateral facial weakness and jaw drop. Truncal and proximal limb weakness was marked. Edrophonium chloride (10 $\mathrm{mg}$ ) given intravenously caused an increase in her power. The blood count, sedimentation rate, electolytes and urea, serum proteins and immunoglobins were normal. Thyroglobulin antibodies were present at $1: 25,000$ but other antibodies including antinuclear factor and striated muscle antibodies were absent. An X-ray of the chest showed healed tuberculosis.

For the first 2 days of her admission, the treatment was not altered. Her condition was stable at the level described above. On the afternoon of the third day she was started on prednisolone $5 \mathrm{mg}$ three times a day in addition to pyridostigmine. Next morning, $18 \mathrm{hr}$ after the first dose of prednisolone, the ptosis 


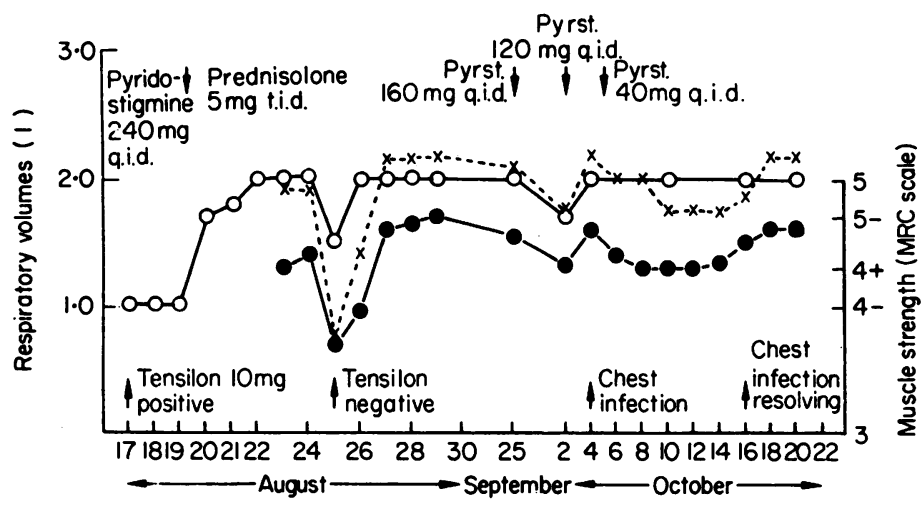

FIG. 1. Shows the muscle strength, $\mathrm{FEV}_{1}$ and FVC plotted against time. Note the rapid improvement following steroid treatment and the acute deterioration thereafter. Respiratory infection caused a fall in $F_{1}$ and FVC but muscle strength remained normal.,$- \mathrm{FEV}_{1}$ (predicted 1.60 ); $\times--\times$, FVC (predicted 2.05); $\bigcirc-0$, muscle power.
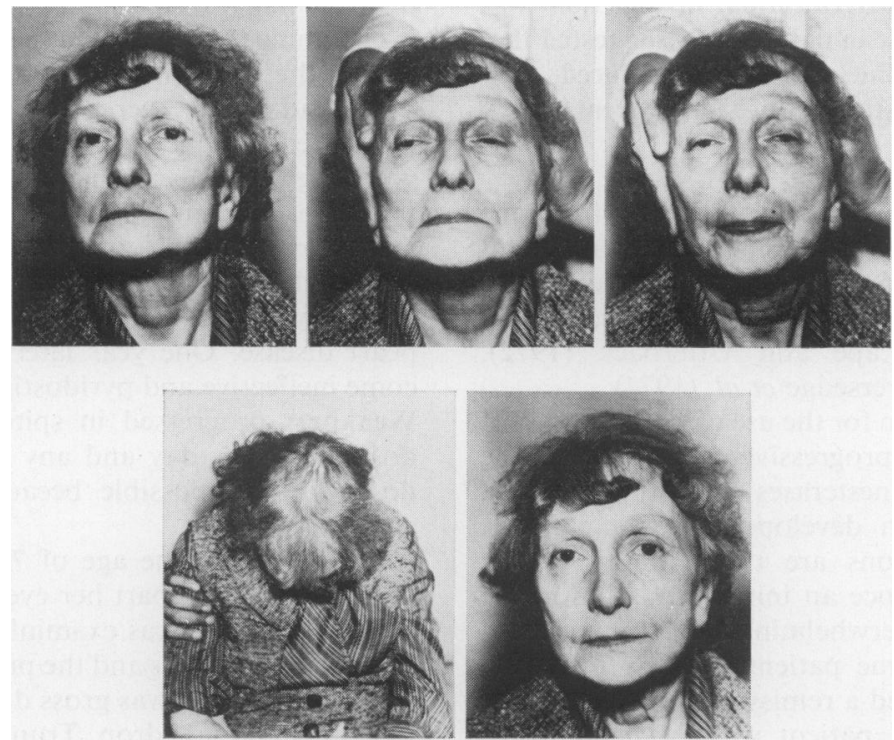

Fig. 2. A typical negative tensilon test-this one was obtained 3 weeks after steroid treatment was started. The tensilon test became positive after pyridostigmine dose was reduced to $120 \mathrm{mg} /$ day.

and the head drop had disappeared; she could stand erect and walk normally without scraping her feet on the ground. Her diplopia disappeared and she could read satisfactorily for the first time in several years. In terms of objective grading her muscle strength had improved from 4- to 5 - (as on MRC scale). Edrophonium chloride $(10 \mathrm{mg})$ given intravenously caused only a minimal improvement in the muscle power. On the following day, no objective weakness was detectable in any muscle group and an injection of edrophonium chloride caused no significant ज़ change.

On the fifth day of treatment with steroids, the patient became weak; there was dysphagia, dysarthria, diffuse muscle weakness and demonstrable fatiguability. She also had constricted pupils, sweaty hands, abdominal cramp and bradycardia. There was a reduction in her respiratory volume, FEV $_{1}$ and FVC (Fig. 1). Edrophonium chloride given intravenously induced a further reduction in 
her muscle power, $\mathrm{FEV}_{1}$ and FVC. Treatment was not altered and her muscle strength gradually improved over the next 2 days. Edrophonium chloride injections consistently caused deterioration in her muscle strength. When she was discharged from the hospital, her muscle strength was normal (grade 5) and $\mathrm{FEV}_{1}$ and FVC were greater than those expected for her age and size. After a 3-week stay at home during which the patient had remained extremely well, she was readmitted for a gradual reduction in the pyridostigmine dose in view of the persistent negative effect of edrophonium on her strength. Its dose was gradually reduced to $120 \mathrm{mg} /$ day; at this point the diarrhoea and abdominal cramp disappeared completely and atropine was stopped. In an attempt to avoid steroid side effects, the dose of prednisolone was halved by administering it only on alternate days. A week later the patient noticed weakness on days when she was not taking prednisolone. This was confirmed by the objective finding of ptosis and weakness of neck extension. Edrophonium chloride tests at this stage were found to cause a marginal improvement in her muscle strength. The dose of prednisolone was changed to $7.5 \mathrm{mg}$ daily and the weakness observed on alternate days disappeared. Anticholinesterases were stopped. The patient has now been maintained on this small dose of prednisolone for 6 months without recurrence of symptoms and without recourse to anticholinesterases.

\section{Discussion}

The most dramatic finding was that the patient's muscular strength improved in a remarkably short time, that is, within $18 \mathrm{hr}$ of the institution of steroid treatment. The other interesting features were that as low a dose as $15 \mathrm{mg} /$ day of prednisolone was able to induce and maintain a remission of her symptoms and that the deterioration following the initial improvement was not as intense as that usually seen following steroid treatment.

There can be little doubt that steroids exerted an acute facilitatory effect on neuromuscular transmission in this patient. That this effect persisted even after weeks of steroid administration is borne out by the fact that when the dose was reduced, the patient experienced weakness on days when she was not given steroids. As in other studies on patients treated with steroids (Cape and Utterback, 1972; Turner, 1974) the present patient developed weakness in the first week of steroid treatment. At no stage was this weakness greater than that observed at the time of her admission. During this phase, she had constricted pupils, sweaty hands and bradycardia. Intravenous edrophonium chloride caused a further decrease in muscular strength and the res- piratory volumes. It may be concluded that the clinical deterioration was in fact a cholinergic crisis. The dose of pyridostigmine which at the beginning of steroid treatment was insufficient-as indicated by the positive edrophonium test-had somehow become excessive following steroid administration. Cape and Utterback (1972) noted that their patients' weakness did not respond to anticholinesterases during this phase. Brunner et al. (1972) found that anticholinesterases either had no effect or in fact caused a further decrease in the muscular strength of their patients at this stage. Turner (1974) and Liversedge et al. (1974), do not comment upon the effect of anticholinesterases during this phase.

In the rat phrenic nerve-diaphragm preparation, prednisolone has been shown experimentally to enhance the release of acetylcholine at the neuromuscular junction; in addition, it has a direct stimulatory effect upon the sarcolemmal membrane (Wilson, Ward and Johns, 1974). An increase in the sensitivity of the sarcolemmal membrane in myasthenics may also occur in fashion similar to that observed in the denervated muscle (Miledi, 1960) since the quantity of acetylcholine released at the neuromuscular junction of these patients is considerably diminished (Elmqvist et al., 1964). Furthermore, steroids may inhibit the binding to acetylcholine receptors of IgG which occurs in myasthenics (Bender et al., 1975); this would make more acetylcholine receptors available thus rendering the sarcolemmal membrane more sensitive to acetylcholine. It is suggested that a combination of the mechanisms described above would account for both the beneficial effect and the subsequent cholinergic crisis in these patients following treatment with steroids.

If these mechanisms do underlie both the beneficial and the hazardous effects of corticosteroid therapy, it would seem that all patients who have a post-therapy cholinergic crisis should eventually benefit from this treatment. This has been the experience of Genkins, Kornfeld and Osserman (1971) who considered that such deterioration initially was a good prognostic feature in 100 myasthenic patients treated with ACTH.

In the present patient the cholinergic crisis improved without reduction of the dose of pyridostigmine initially, although later the drug was successfully withdrawn completely. This suggests that the heightened sensitivity of the sarcolemmal membrane to acetylcholine is rapidly reversible, but the authors know of no experimental observations to support this concept.

If further studies show that deterioration following corticosteroid therapy can be ascribed to excessive cholinergic activity in all or most patients, the wisdom of giving large doses of steroids (or ACTH) 
in combination with the usual, large doses of anticholinesterases would have to be reconsidered.

\section{Acknowledgments}

We are grateful to Dr C. W. H. Havard and to Dr J. Newsom Davis for their helpful criticisms.

\section{References}

Bender, A.N., Ringel, S.P., Engel, W.K., Daniels, M.P. \& VOGEL, Z. (1975) Myasthenia gravis: a serum factor blocking acetylcholine receptors of the human neuromuscular junction. Lancet, i, 607.

Brunner, N.G., Namba, T. \& Grob, D. (1972) Corticosteroids in the management of severe, generalized myasthenia gravis. Neurology, 22, 603.

CAPE, C.A. \& UTTERBACK, R.A. (1972) Maintenance adrenocorticotropic hormone (ACTH) treatment in myasthenia gravis. Neurology, 22, 1160.
Elmovist، R., Hoffman, W.W., Kugelberg, J., Quastel, D.M.J. (1964) Electrophysiological investigation of neuromuscular transmission in myasthenia gravis. Journal of Physiology, 174, 417.

Genkins, G., Kornfeld, P. \& Osserman, K.E. (1971) The use of ACTH and corticosteroids in myasthenia gravis. Annals of the New York Academy of Sciences, 183, 369.

Liversedge, L.A., Yuill, G.M., Wilkinson, I.M. \& Hughes, R. (1974) Benefit from adrenocorticotrophin in myasthenia gravis. Journal of Neurology, Neurosurgery and Psychiatry, 37, 412.

Miledi, R. (1960) The acetylcholine sensivity of frog muscle fibres after complete or partial denervation. Journal of Physiology, 151, 1.

Turner, A. (1974) Myasthenia gravis. Proceeaings of the Royal Society of Medicine, 67, 763.

Wilson, R.W., WARD, M.D. \& Johns, T.R. (1975) Corticosteroids: a direct effect at the neuromuscular junction Neurology, 24, 1091.

\title{
Pseudomembranous enterocolitis complicating ampicillin therapy
}

\author{
L. READ \\ B.Sc., M.B., B.S. \\ J. R. Cove-Smith* \\ M.A., M.R.C.P. \\ Departments of Orthopaedic Surgery and ${ }^{*}$ General Medicine, City Hospital, Nottingham
}

\begin{abstract}
Summary
A case of fatal pseudomembranous enterocolitis associated with ampicillin therapy is described and possible mechanisms for its production discussed.
\end{abstract}

\section{Introduction}

The association of pseudomembranous enterocolitis with antibiotic therapy is well recognized (Leading Article, 1975; Tedesco, Barton and Alpers, 1974). Most reports have been associated with clindamycin or lincomycin (Tedesco et al., 1974; Crapp et al., 1975), but tetracycline (Klotz, Palmer and Kirsner, 1953), chloramphenicol (Reiner, Schlesinger and Miller, 1952) and ampicillin (Schapiro and Newman, 1973; Keating et al., 1974) have also been incriminated. A case is now reported of fatal pseudomembranous enterocolitis associated with ampicillin and cloxacillin therapy.

\section{Case report}

A 73-year-old woman with a long history of arthritis underwent a Manchester condylar arthroplasty of the right knee in September 1975. Rheuma-

Correspondence: Dr J. R. Cove-Smith, Renal Unit, City Hospital, Hucknall Road, Nottingham NG5 1PB. toid arthritis had been diagnosed in 1967 when she had a successful left hip replacement, and since then she had been taking prednisolone $2.5 \mathrm{mg}$ b.d. Cover for the operation included hydrocortisone $100 \mathrm{mg} 6$ hourly, ampicillin $250 \mathrm{mg}$ 6-hourly and cloxacillin $500 \mathrm{mg}$ 6-hourly. Both antibiotics were continued for 23 days. Initial postoperative progress was uneventful, but on the seventh day she developed dysuria and pyrexia; urine culture grew Klebsiella aerogenes resistant to ampicillin. Co-trimoxazole produced an immediate hypotensive reaction, with an itchy erythematous rash and generalized oedema; it was withdrawn after one dose. Nitrofurantoin $100 \mathrm{mg}$ q.i.d. for 10 days cleared the infection, and subsequent urine and blood cultures were not infected. From the tenth postoperative day diarrhoea and vomiting became a problem. The vomiting stopped when the ampicillin and cloxacillin were given intramuscularly, but loose watery stools continued up to six times a day with faecal incontinence at night. The wound edges were necrotic but clean. The antibiotics were stopped and intravenous fluids started on the twenty-third postoperative day. Seven days later she developed a pyrexia and slime was noticed in the motions. Sigmoidoscopy showed numerous 